\title{
Yttrium Y-90 Ibritumomab Tiuxetan
}

National Cancer Institute

\section{Source}

National Cancer Institute. Yttrium Y-90 Ibritumomab Tiuxetan. NCI Thesaurus. Code C1812.

A radioimmunotherapeutic agent consisting of a murine monoclonal anti-CD20 antibody (ibritumomab) linked by the chelator tiuxetan to the radioisotope yttrium-90 (Y 90).

Yttrium Y 90 ibritumomab tiuxetan binds to and specifically delivers beta radiation to CD20-expressing tumor cells, thereby minimizing the systemic effects of radiation. 